\title{
Factors associated with decreased bone mineral density in postmenopausal women with schizophrenia
}

\author{
Ying Liang' \\ Jian Huang' \\ Jing-bin Tian ${ }^{2}$ \\ Yuan-yuan $\mathrm{Cao}^{2}$ \\ Guo-ling Zhang ${ }^{2}$ \\ Chun-gang Wang ${ }^{2}$ \\ Ying $\mathrm{Cao}^{2}$ \\ Jian-rong $\mathrm{Li}^{2}$
}

'National Clinical Research Center for Mental Disorders, Peking University Sixth Hospital, Institute of Mental Health, and the Key Laboratory of Mental Health, Ministry of Health, Peking University, Beijing, People's Republic of China; ${ }^{2}$ Psychiatry Department, Changping District Hospital of Integrated Chinese and Western Medicine, Beijing, People's Republic of China
Correspondence: Ying Liang

National Clinical Research Center for Mental Disorders, Peking University Sixth Hospital, Institute of Mental Health, and the Key Laboratory of Mental Health, Ministry of Health, Peking University, Huayuanbeilu Street 5I, Haidian District, Beijing I0019I, People's Republic of China Tel +861082801903

Fax +86 I0 82801908

Email liangying1980@bjmu.edu.cn
This article was published in the following Dove Press journal:

Clinical Interventions in Aging

17 February 2016

Number of times this article has been viewed

Objective: This study examined the risk factors for decreased bone mineral density (BMD) in postmenopausal women with schizophrenia.

Methods: Cluster sampling method was adopted in this large-sample, cross-sectional study. A total of 219 postmenopausal female inpatients with schizophrenia were selected and interviewed in Beijing. The average age of the patients was $60.4 \pm 7.0$ years. Clinical assessment instruments included the Positive and Negative Syndrome Scale (PANSS) and a questionnaire with detailed general information and disease-related investigations. Laboratory measurements included prolactin (PRL), estradiol, progesterone, thyroid stimulating hormone, FT3, and FT4. BMD testing was performed by dual-energy X-ray absorptiometry.

Results: The prevalence of osteoporosis or osteopenia was $66.2 \%(n=145)$. Decreased BMD was associated with age, illness duration, therapeutic dose (equivalent chlorpromazine dose), treatment duration, PANSS-negative scores, body mass index (BMI), daily exercises (min/d), drinking (unit/wk), PRL, and estradiol. Multiple logistic regression analysis revealed that age, treatment duration, PANSS-negative score, BMI, and PRL were significantly associated with decreased BMD.

Conclusion: Prevalence of BMD loss was higher in Chinese postmenopausal women with schizophrenia compared to the normal BMD group. A combination of demographic and clinical factors play important roles in determining decreased BMD, including older age, longer treatment duration, more PANSS-negative scores, higher BMI, and higher PRL level.

Keywords: schizophrenia, bone density, risk factors

\section{Introduction}

The risk of medical comorbidities in patients with schizophrenia is significantly higher than that in the general population, ${ }^{1}$ and osteoporosis is one of these. Numerous studies have consistently revealed that patients with schizophrenia have significantly lower bone mineral density (BMD) compared with their peers of the same sex in the general population. ${ }^{2,3}$ This issue has approximately doubled the risk of femoral neck fractures in patients with schizophrenia compared with the normal population, ${ }^{4,5}$ as well as increased rates of morbidity and mortality, which place a heavy economic burden on society.

The etiology of decreased BMD in patients with schizophrenia remains obscure. In recent years, the influence of hyperprolactinemia caused by antipsychotic drugs on BMD has continuously gained more attention from researchers. Numerous studies have found that most patients with chronic schizophrenia on long-term antipsychotic medications (especially "prolactin [PRL]-elevating" drugs) revealed a significantly 
increased risk of decreased BMD or osteoporosis. ${ }^{6,7}$ However, other studies have obtained the opposite result, ${ }^{8}$ which implies that there might be some other factors that affect the BMD of patients with schizophrenia. Indeed, since negative symptoms and social functions decline, schizophrenic illness shows numerous unhealthy lifestyle behaviors that can increase the risk of osteoporosis, which include limited exercise, lack of exposure to sunshine, poor nutrition, smoking, and alcoholism. ${ }^{9}$ However, reports on the effects of unhealthy lifestyle on BMD in patients with schizophrenia are very rare. Merely two studies have found that body mass index (BMI) and smoking may be associated with bone loss in patients with schizophrenia. ${ }^{6,10}$

However, to date, no study has focused on bone loss in patients with schizophrenia in the People's Republic of China, where there are many different sociodemographic situations compared to other countries. Therefore, examining the factors associated with decreased BMD in patients with schizophrenia is of great importance in the People's Republic of China.

The main aim of our study was to explore the related risk factors for decreased BMD in postmenopausal women with schizophrenia.

\section{Methods}

\section{Settings and subjects}

In this large-sample, cross-sectional study, cluster sampling method was adopted, and subjects were recruited from a group of patients with schizophrenia confined at the Changping District Hospital of Integrated Chinese and Western Medicine, Beijing, since April 2014. Inclusion criteria were as follows: 1) female patients with a diagnosis of schizophrenia according to the Diagnostic and Statistical Manual of Mental Disorders, fourth edition diagnostic criteria and 2) all women $>52$ years old, who were confirmed to be postmenopausal by a gynecologist as having no menstruation for at least 2 years. Exclusion criteria were as follows: 1) patients who are taking any medication known to be risk factors for BMD decline such as antidepressant and anticonvulsant drugs; 2) patients who have medical disorders known to be risk factors for BMD decline such as alcohol dependence and eating disorder; and 3) patients who have a family history of osteoporosis. From the 692 original samples obtained, 219 patients met our inclusion criteria and participated in this study. The mean age of the patients was $60.4 \pm 7.0$ years.

\section{Clinical assessment}

Symptom severity was rated using the Positive and Negative Syndrome Scale (PANSS). ${ }^{11}$ The questionnaire included detailed information regarding the general information and disease-related investigations relevant to $\mathrm{BMD}$, including name, age, sex, date of birth, birth place, education, vocation, marital status and address, age of onset, illness duration, previous disease and drug histories, smoking and alcohol history, serum calcium, serum phosphorus, fasting blood glucose, daily exercises, female menstrual history and current drug administration list, and equivalent chlorpromazine (CPZ) dose of current antipsychotic drug, ${ }^{12}$ and was completed for each participant.

\section{Laboratory assessments}

Venous blood specimens were collected from patients at 7 to 9 am in the next morning, after fasting for 8-12 hours, by specially trained research nurses. The blood sample $(5 \mathrm{~mL})$ was centrifuged at 3,000 rpm for 5 minutes after 4 hours of standing at $4^{\circ} \mathrm{C}$. Approximately $1.5 \mathrm{~mL}$ of serum was subsequently separated and carefully transferred to a disposable cup and then stored at an ultra-low $\left(-80^{\circ} \mathrm{C}\right)$ temperature for subsequent analysis. Before the test, the stored serum samples were removed from the $-80^{\circ} \mathrm{C}$ freezer, placed in a $4^{\circ} \mathrm{C}$ refrigerator to thaw, and the test was finished within 48 hours. On the day of the test, samples were maintained undisturbed at room temperature until the temperature was balanced. All the tests were accomplished in the clinical laboratory of Changping District Hospital of Integrated Chinese and Western Medicine, Beijing. PRL, estradiol (E2), progesterone, thyroid stimulating hormone, free triiodothyronine (FT3), and free thyroxine (FT4) levels were measured using chemiluminescence with an automated chemiluminescence analyzer ADVIA Centaur (Siemens AG, Munich, Germany).

\section{BMD assessment}

BMD testing was performed by dual-energy X-ray absorptiometry (GE Healthcare Bio-Sciences Corp., Piscataway, NJ, USA) in the lumbar spine (L1-L4) and femoral neck. Quality assurance was performed before the measurement; all tests were two-tailed and the random error of replication was $<1 \%$. The scan image of bone surface density is defined as $1.001 \mathrm{~g} / \mathrm{cm}^{2}$, and results can be calculated automatically. $T$-score values, which compare BMD with standardized bone mass for ethnic-, sex-, and age-specific groups, were used in the subsequent analysis. Osteoporosis is defined as a BMD value that falls at least 2.5 standard deviation (SD) below these standardized values, and osteopenia is defined as a BMD value that falls $1 \mathrm{SD}$ below normative values. A BMD value higher than $-1.0 \mathrm{SD}$ means normal.

\section{Statistical analysis}

Data were analyzed with SPSS 17.0 (Statistical Package for Windows), and results are expressed as mean \pm standard 
Table I Comparison of demographic and clinical characteristics between abnormal and normal BMD groups of patients with schizophrenia

\begin{tabular}{|c|c|c|c|c|c|c|}
\hline & \multicolumn{2}{|c|}{ Abnormal BMD group $(n=\mid 45)$} & \multicolumn{2}{|c|}{ Normal BMD group $(n=74)$} & \multicolumn{2}{|c|}{ Statistics } \\
\hline & Mean/median & SD/(QI, Q3) & Mean/median & SD/(QI, Q3) & $T / Z$ & $P$-value \\
\hline Age & 62.1 & 7.2 & 59.1 & 5.2 & 3.49 & 0.001 \\
\hline Illness duration (years) & 30.9 & 10.3 & 28.2 & 9.4 & 1.88 & 0.061 \\
\hline Current therapeutic dose (CPZ) & 450.0 & $(359.1,612.5)$ & 400.0 & $(272.9,612.5)$ & -2.31 & 0.021 \\
\hline Treatment duration (years) & 28.9 & 10.5 & 24.1 & 9.2 & 3.46 & 0.001 \\
\hline Total PANSS score & 52.2 & 13.2 & 51.7 & 13.8 & 0.26 & 0.798 \\
\hline PANSS positive & 12.8 & 4.3 & 13.5 & 5.5 & -0.98 & 0.327 \\
\hline PANSS negative & 15.2 & 4.2 & 11.7 & 2.8 & 7.26 & 0.000 \\
\hline $\mathrm{BMI}, \mathrm{kg} / \mathrm{m}^{2}$ & 23.5 & 3.8 & 25.6 & 4.6 & -3.52 & 0.001 \\
\hline Calcium & 2.2 & 0.1 & 2.2 & 0.1 & 0.11 & 0.909 \\
\hline Phosphorus & 1.1 & 0.2 & 1.2 & 0.2 & -1.45 & 0.149 \\
\hline FBG & 4.8 & 1.2 & 5.1 & 1.8 & -1.15 & 0.252 \\
\hline Exercises per week (minutes) & 267.6 & 109.9 & 310.2 & 129.9 & -2.55 & 0.012 \\
\hline No of cigarette smoked per day & 0 & $(0,10)$ & 0 & $(0,10)$ & -0.09 & 0.928 \\
\hline Drinking (unit/wk) & 1.0 & $(0,1.0)$ & 0 & $(0,1.0)$ & -2.95 & 0.003 \\
\hline TSH & 2.9 & 2.2 & 3.9 & 5.8 & -1.46 & 0.147 \\
\hline FT3 & 2.4 & 0.4 & 2.3 & 0.6 & 1.73 & 0.085 \\
\hline FT4 & 1.0 & 0.8 & 1.2 & 1.5 & -1.39 & 0.168 \\
\hline Prolactin & 38.2 & 26.4 & 20.4 & 10.2 & 7.15 & 0.000 \\
\hline Estradiol & 25.5 & 14.6 & 31.9 & 21.3 & -2.29 & 0.024 \\
\hline Progesterone & 0.8 & 1.1 & I.I & 2.0 & -1.28 & 0.202 \\
\hline
\end{tabular}

Abbreviations: BMD, bone mineral density; SD, standard deviation; CPZ, chlorpromazine; PANSS, Positive and Negative Syndrome Scale; BMI, body mass index; FBG, fasting blood glucose; wk, week; TSH, thyroid stimulating hormone; FT3, free triiodothyronine; FT4, free thyroxine; T/Z, T-score/Z-score.

deviation. Independent $t$-tests (two-tailed) and rank-sum test were used to identify significant differences between groups, and $\chi^{2}$ tests were performed to analyze cross-tabulations wherever appropriate. Multiple logistic regression analyses were performed to identify correlations between variables that hypothesized a priori to be correlated with decreased BMD. A $P$-value $<0.05$ was considered significant.

\section{Ethical considerations}

According to the Guideline for Good Clinical Practice of the International Conference on Harmonisation, the research protocol and the informed consent form was approved by Institutional Review Boards of Peking University's Institute of Mental Health. All subjects or their legal guardians provided written consent after understanding the aims of this study and were included in this study.

\section{Results}

The prevalence of osteoporosis or osteopenia among the 219 study participants was $66.2 \%(n=145) . T$ scores of the lumbar spine (L1-L4), left femoral neck, and right femoral neck were $-0.7(-1.8,0.4),-0.9(-1.7,0.1)$, and $-0.8(-1.8,0)$, respectively.

Patients with osteoporosis and osteopenia were assigned into the BMD abnormal group, while the remaining patients were assigned into the normal group. Differences in age, illness duration, therapeutic dose $(\mathrm{CPZ})$, treatment duration, PANSS-negative scores, BMI, daily exercises (min/d), drinking (unit/wk), PRL, and estradiol between the two groups were statistically significant. Details are shown in Table 1. The multiple logistic regression analysis showed that age, treatment duration, therapeutic dose (CPZ), PANSS-negative scores, BMI, exercises per week (minutes), and PRL were significantly associated with decreased BMD. Details are shown in Table 2.

\section{Discussion}

Our study revealed that $66.2 \%$ of Chinese postmenopausal women with schizophrenia exhibited decreased BMD. This figure is similar to that reported in other Asian countries, ${ }^{13}$ but is significantly higher than that in European countries. ${ }^{6}$

Table 2 Factors associated with abnormal BMD (multiple logistic regression analysis)

\begin{tabular}{lllllll}
\hline Factor & B & SE & $\begin{array}{l}\text { Wald } \\
\text { test }\end{array}$ & P-value & $\begin{array}{l}\text { Odds } \\
\text { ratio }\end{array}$ & $\mathbf{9 5 \%} \mathbf{C l}$ \\
\hline Age & 0.10 & 0.03 & 9.17 & 0.002 & $\mathrm{I} . \mathrm{II}$ & $\mathrm{I} .04-\mathrm{I} .19$ \\
$\begin{array}{l}\text { Treatment } \\
\text { duration }\end{array}$ & 0.08 & 0.03 & 5.87 & 0.015 & $\mathrm{I} .08$ & $\mathrm{I} .02-\mathrm{I} . \mathrm{I5}$ \\
$\begin{array}{l}\text { PANSS negative } \\
\text { BMI, kg/m }\end{array}$ & 0.29 & 0.06 & 26.59 & 0.000 & $\mathrm{I} .34$ & $\mathrm{I} .20-\mathrm{I} .50$ \\
Prolactin & $-0.1 \mathrm{I}$ & 0.05 & 5.16 & 0.023 & 0.89 & $0.8 \mathrm{I}-0.98$ \\
\hline
\end{tabular}

Abbreviations: $\mathrm{BMD}$, bone mineral density; SE, standard error; $\mathrm{Cl}$, confidence interval; PANSS, Positive and Negative Syndrome Scale; BMI, body mass index. 
A study reported that BMD of postmenopausal female patients decreased faster and the prevalence of decreased BMD was higher in Asian populations, compared to that in Caucasians. ${ }^{14}$ This difference might be due to racial differences and other environmental protective factors such as a higher BMI in white women. ${ }^{15}$

The present study revealed that decreased BMD in patients with schizophrenia is associated with hyperprolactinemia and lower estradiol, which implies that decreased BMD is associated with hyperprolactinemia caused by antipsychotics, and lower secondary sex hormone levels. This result has been repeatedly demonstrated in animal experiments. ${ }^{16,17}$ However, results of previous clinical studies have been inconsistent. ${ }^{6-9,18-20}$ The reasons may be related to the following factors: first, the sample size of the previous study was small; second, majority of the methods of grouping were too simple, which only divide the current antipsychotic treatment of schizophrenia into "PRL-raising drug group vs PRL-sparing drug group" or "high dose group vs low dose group"; third, almost all studies did not give a clear definition of other psychiatric medications, which may affect BMD (eg, antidepressants and anticonvulsants). The present study used a larger sample, included postmenopausal women with solely schizophrenia, excluded interferences of antidepressant or anticonvulsant drugs, and considered various lifestyles that sufficiently affect BMD.

Furthermore, our study revealed that decreased BMD in patients with schizophrenia is related to the long treatment duration of antipsychotic drugs, which was not reported in previous studies. This might be related to the large sample size and the sole inclusion of postmenopausal women with schizophrenia in this study. This also confirms that antipsychotics can cause decreased BMD, which warns clinicians to notice the risk of antipsychotic drugs in causing decreased BMD and fractures. Recently, two large studies ${ }^{21,22}$ have consistently found that the use of antipsychotic drugs affects the prevalence of fractures, and the treatment duration of antipsychotics was positively correlated with the risk of fracture.

In addition, this study found that BMI is a protective factor in BMD, which is consistent with previous studies. ${ }^{11}$ Weight is a mechanical load factor, which has different effects in different parts of BMD, reflecting various parts to withstand various mechanical loads. The greater weight of the bones would bear a greater load and have a higher bone density. In addition, negative symptoms are a risk factor for bone density, which is consistent with previous studies. ${ }^{19}$ This may be due to the negative symptoms of schizophrenia, which would more likely lead to lack of exercise, reduction to sun exposure, and calcium and vitamin $\mathrm{D}$ deficiency caused by nutritional deficiencies.

Osteoporosis is a kind of disease that causes serious harm to public health. This study found that the prevalence of BMD loss was higher in Chinese postmenopausal women with schizophrenia, compared to the normal BMD group. In addition, a combination of demographic and clinical factors play important roles in determining decreased BMD, including older age, longer treatment duration, more PANSSnegative scores, higher BMI, and higher PRL levels.

These findings suggest that clinical psychiatrists should pay more attention in monitoring BMD in patients with schizophrenia, especially older female patients with longterm medications. In addition, clinical intervention for patients with decreased BMD can be carried out based on the following aspects: 1) the use of drugs causing minimal influence on PRL, so as to avoid hyperprolactinemia that could result in bone loss; 2) enhance the improvement and treatment of negative symptoms of schizophrenia, thereby increasing patient activity, sunlight exposure, and enough calcium and vitamin D intake; 3) reduce malnutrition in patients with schizophrenia caused by lower-than-normal BMI phenomenon, thereby keeping the patient in the normal weight range, which may also have a protective effect on bone loss.

In the present study, despite the larger sample size than the previous study, the impact of unhealthy lifestyles that decrease BMD in schizophrenia and the influence of antipsychotics were fully considered. However, inevitably, there are some methodological shortcomings. This is a cross-sectional study, rather than a prospective study, and only factors that may be associated with decreased BMD in schizophrenia were found, and a causal relationship could not be determined. Large-sample, prospective studies are needed in the future to further confirm the results of this study.

\section{Conclusion}

Prevalence of BMD loss was higher in Chinese postmenopausal women with schizophrenia compared to the normal BMD group. A combination of demographic and clinical factors play important roles in determining decreased BMD, including older age, longer treatment duration, more PANSSnegative scores, higher BMI, and higher PRL level.

\section{Disclosure}

The authors report no conflicts of interest in this work. 


\section{References}

1. Hert DE, Correll M, Bobes CU, et al. Physical illness in patients with severe mental disorders. I. Prevalence, impact of medications and disparities in health care. World Psychiatry. 2011;10:52-77.

2. Partti K, Heliövaara M, Impivaara O, et al. Skeletal status in psychotic disorders: a population-based study. Psychosom Med. 2010;72: 933-940.

3. Renn JH, Yang NP, Chueh CM, Lin CY, Lan TH, Chou P. Bone mass in schizophrenia and normal populations across different decades of life. BMC Musculoskelet Disord. 2009;10:1.

4. Bolton JM, Metge C, Lix L, Prior H, Sareen J, Leslie WD. Fracture risk from psychotropic medications: population-based analysis. J Clin Psychopharmacol. 2008;28:384-391.

5. Howard L, Kirkwood G, Leese M. Risk of hip fracture in patients with a history of schizophrenia. Br J Psychiatry. 2007;190:129-134.

6. Meaney AM, Smith S, Howes OD, O'Brien M, Murray RM, O'Keane V. Effects of long-term prolactin-raising antipsychotic medication on bone mineral density in patients with schizophrenia. Br J Psychiatry. 2004; 184:503-508.

7. Becker D, Liver O, Mester R, Rapoport M, Weizman A, Weiss M. Risperidone, but not olanzapine, decreases bone mineral density in female premenopausal schizophrenia patients. J Clin Psychiatry. 2003;64: 761-766.

8. Sugawara N, Yasui-Furukori N, Fujii A, et al. No association between bone mass and prolactin levels among patients with schizophrenia. Hum Psychopharmacol. 2011;26:596-601.

9. Howes OD, Wheeler M, Meaney AM, et al. Bone mineral density and its relationship to prolactin levels in patients taking antipsychotic treatment. J Clin Psychopharmacol. 2005;25(3):259-261.

10. Vancampfort D, Knapen J, Probst M, Scheewe T, Remans S, De Hert M. A systematic review of correlates of physical activity in patients with schizophrenia. Acta Psychiatr Scand. 2012;125:352-362.

11. Kay SR, Fiszbein A, Opler LA. The positive and negative syndrome scale (PANSS) for schizophrenia. Schizophr Bull. 1987;13:261-276.
12. Woods SW. Chlorpromazine equivalent doses for the newer atypical antipsychotics. J Clin Psychiatry. 2003;64:663-667.

13. Jung DU, Kelly DL, Oh MK, et al. Bone mineral density and osteoporosis risk in older patients with schizophrenia. J Clin Psychopharmacol. 2011;31:406-410.

14. Xiaoguang C, Dingchao Y, Zhuo Z. The incidence of Chinese women age related bone mineral density, bone loss, osteoporosis and databasemulticenter cooperative projects. Chin J Osteoporosis. 2008;14(4): 221-228.

15. Ross PD, He YE, Yates AJ, et al. Body size accounts for most differences in bone density between Asian and Caucasian women. Calcif Tissue Int. 1996;59(339):2343.

16. Kunimatsu T, Kimura J, Funabashi H, Inoue T, Seki T. The antipsychotics haloperidol and chlorpromazine increase bone metabolism and induce osteopenia in female rats. Regul Toxicol Pharmacol. 2010; 58(3):360-368.

17. Sackett G, Unis A, Crouthamel B. Some effects of risperidone and quetiapine on growth parameters and hormone levels in young pigtai macaques. J Child Adolesc Psychopharmacol. 2010;20(6):489-493.

18. Lin $\mathrm{CH}$, Huang $\mathrm{KH}$, Chang YC, et al. Clozapine protects bone mineral density in female patients with schizophrenia. Int J Neuropsychopharmacol. 2012;15(7):897-906.

19. Lee TY, Chung MY, Chung HK, Choi JH, Kim TY, So HS. Bone density in chronic schizophrenia with long-term antipsychotic treatment: preliminary study. Psychiatry Investig. 2010;7:278-284.

20. O'Keane V, Meaney AM. Antipsychotic drugs: a new risk factor for osteoporosis in young women with schizophrenia? J Clin Psychopharmacol. 2005;25:26-31.

21. Vestergaard P, Rejnmark L, Mosekilde L. Anxiolytics, sedatives, antidepressants, neuroleptics and the risk of fracture. Osteoporos Int 2006; 17:807-816.

22. Hugenholtz GW, Heerdink ER, van Staa TP, Nolen WA, Egberts AC. Risk of hip/femur fractures in patients using antipsychotics. Bone. $2005 ; 37: 864-870$.
Clinical Interventions in Aging

\section{Publish your work in this journal}

Clinical Interventions in Aging is an international, peer-reviewed journal focusing on evidence-based reports on the value or lack thereof of treatments intended to prevent or delay the onset of maladaptive correlates of aging in human beings. This journal is indexed on PubMed Central, MedLine,

\section{Dovepress}

CAS, Scopus and the Elsevier Bibliographic databases. The manuscript management system is completely online and includes a very quick and fair peer-review system, which is all easy to use. Visit http://www.dovepress. com/testimonials.php to read real quotes from published authors. 